\title{
Correction to: A cluster of disseminated small cortical lesions in MELAS: its distinctive clinical and neuroimaging features
}

\author{
Yu Hongo ${ }^{1}$ Juntaro Kaneko ${ }^{1}$ Hiroki Suga ${ }^{1}$ - Daisuke Ishima ${ }^{1}$ - Eiji Kitamura ${ }^{1} \cdot$ Tsugio Akutsu $^{1} \cdot$ Yuya Onozawa $^{2}$. \\ Naomi Kanazawa ${ }^{1} \cdot$ Tomohide Goto $^{3} \cdot$ Kazutoshi Nishiyama $^{1} \cdot$ Takahiro lizuka $^{1}$ (1)
}

Published online: 9 April 2019

๑) Springer-Verlag GmbH Germany, part of Springer Nature 2019

\section{Correction to: Journal of Neurology https://doi.org/10.1007/s00415-019-09283-3}

The author would like to correct the errors in the publication of the original article. The corrected details are given below for your reading.

In "Clinical features and neuroimaging patterns of SLEs" section, sixth sentence of the fifth paragraph should as:

In patient 11, brain MRIs obtained 16 weeks after the symptoms onset showed extensive gadolinium enhancement along the gyri of the affected cortexes, indicating not only focal disruption of the BBB, but also same subacute stage of all disseminated lesions (Fig. $2 \mathrm{~g}-\mathrm{i}$ ).
The original article can be found online at https://doi.org/10.1007/ s00415-019-09283-3.

Takahiro Iizuka

takahiro@med.kitasato-u.ac.jp

1 Department of Neurology, Kitasato University School of Medicine, 1-15-1 Kitasato, Minami-ku, Sagamihara, Kanagawa 252-0374, Japan

2 Department of Clinical Laboratory, Kitasato University Hospital, Sagamihara, Japan

3 Department of Neurology, Kanagawa Children's Medical Center, Yokohama, Japan
In "Discussion" section, ninth sentence of the third paragraph should as:

We also reported a patient with $\mathrm{m} .617 \mathrm{G}>\mathrm{A}$ who developed recurrent embolic strokes associated with carotid artery stenosis as a phenotype of macroangiopathy [19]. 